\title{
A refined classification approach by integrating Landsat Operational Land Imager (OLI) and RADARSAT-2 imagery for land-use and land-cover mapping in a tropical area
}

\begin{abstract}
Producing accurate land-use and land-cover (LULC) mapping is a long-standing challenge using solely optical remote-sensing data, especially in tropical regions due to the presence of clouds. To supplement this, RADARSAT images can be useful in assisting LULC mapping. The fusion of optical and active remote-sensing data is important for accurate LULC mapping because the data from different parts of the spectrum provide complementary information and often lead to increased classification accuracy. Also, the timeliness of using synthetic aperture radar (SAR) fills information gaps during overcast or hazy periods. Therefore, this research designed a refined classification procedure for LULC mapping for tropical regions. Determining the best method for mapping with a specific data source and study area is a major challenge because of the wide range of classification algorithms and methodologies available. In this study, different combinations and the potential of Landsat Operational Land Imager (OLI) and RADARSAT-2 SAR data were evaluated to select the best procedure for LULC classification. Results showed that the best filter for SAR speckle reduction is the $5 \times 5$ enhanced Lee. Furthermore, image-sharpening algorithms were employed to fuse Landsat multispectral and panchromatic bands and subsequently these algorithms were analysed in detail. The findings also confirmed that Gram-Schmidt (GS) performed better than the other techniques employed. Fused Landsat data and SAR images were then integrated to produce the LULC map. Different classification algorithms were adopted to classify the integrated Landsat and SAR data, and the maximum likelihood classifier (MLC) was considered the best approach. Finally, a suitable classification procedure was designed and proposed for LULC as mapping in tropical regions based on the results obtained. An overall accuracy of $98.62 \%$ was achieved from the proposed methodology. The proposed methodology is a useful tool in industry for mapping purposes. Additionally, it is also useful for researchers, who could extend the method for different data sources and regions.
\end{abstract}

Keyword: Remote-sensing; Landsat Operational Land Imager (OLI); RADARSAT-2 
